\title{
REPLY TO BJØRDAL
}

\author{
ZACH WEBER \\ University of Melbourne
}

§1. In Bjørdal (2010), Bjørdal presents a paraconsistent set theory in which $\forall x(x \neq x)$ is a theorem. The author rightly claims that, while not trivializing (in the sense of proving everything), results like this are to be avoided. The set theory presented in Bjørdal (2010) is based on that of Weber (2010b), but with an introduced definition of identity - which is used, in effect, as a new axiom. With this added notion of identity, the non-self-identity of every object does in fact obtain; and so the set theory presented by Bjørdal is inadequate. ${ }^{1}$

Notably, the form of identity so used is not a part of Weber (2010b), which is a paraconsistent set theory in the relevant logic $T L Q$. Contrary to impressions, ${ }^{2}$ what Bjørdal has shown is that a particular form of identity is not supported in the suggested framework of Weber (2010b). Bjørdal has given evidence for the practical maximality of paraconsistent set theory in $T L Q$. As it was intended to be, this system would be the strongest possible that both endorses a full naive comprehension axiom, while at the same time avoiding triviality. Adding anything more, however apparently innocuous, however wafer thin, will end in disaster.

In this note I show three things. First, I emphasize that the trivializing identity principle is not a part of the system in Weber (2010b). Then I explain why it should not be, either, and not only for ad hoc reasons. One can, of course, introduce a connective $=$ in the manner of Bjørdal (2010), and then derive untoward results. (The same can be said of 'tonk'.) The substantial point is whether $=$, so defined, is identity. We will see that, in the context of a relevant, paraconsistent solution to the naive set theory problem, it is not. Finally, I recall what principles of identity are in the system, and argue that these are more than adequate.

\$2. The set theory of Weber (2010b) is couched in an intensional logic, with relevant implication connective $\rightarrow$, and is intended to solve the naive set theory problem, characterized by two criteria:

- Endorse the axioms of comprehension and extensionality:

AXIOM 1. $x \in\{z: A\} \leftrightarrow A$.

Received: May 26, 2010

1 Though we might mention that the nontriviality of relevant arithmetic has been established with a two element model in which everything is non-self-identical (Mortensen, 1995).

2 The author writes: "We do not show that the proposed system [of Weber (2010b)] is trivial in the stated sense, but suggest it is inadequate in the sense of proving theorems which one should not want as theorems even in a paraconsistent framework" (Bjørdal, 2010). 
AXIOM 2. $x=y \leftrightarrow \forall z(z \in x \leftrightarrow z \in y)$.

- Prove as many mathematically interesting theorems as possible, including both orthodox, foundational facts like Peano's postulates as well as unorthodox, inconsistent facts like the Burali-Forti paradox, without proving absolute nonsense.

Straightforwardly, then, any logical principle that falls afoul of these criteria, those that result in noise like $\forall x(x \neq x)$, is not part of a solution to the naive set problem. 'Axiom chopping' in this context is like noticing that multiplication of quaternions is associative but not commutative, while for octonions it is neither. The strategy is principled. But we can give even better reasons for our choices.

In what follows, we must observe the distinction between an inference, marked with $\vdash$, and an implication, marked with $\rightarrow$. The latter preserves relevance; the former does not. Critically,

$$
\text { if } A \leftrightarrow B \text {, then } \neg A \leftrightarrow \neg B \text {; }
$$

but

$$
A \dashv \vdash B \text { does not necessarily yield } \neg A \dashv \vdash \neg B \text {, or vice versa. }
$$

A very effective technique in working to solve the naive set theory problem is to determine which of $\vdash, \rightarrow$ are appropriate in which places.

§3. The system of Weber (2010b) is a proposed solution to the naive set theory problem. Bjørdal adds to it the following: ${ }^{3}$

$$
\vdash x=y \rightarrow(A x \rightarrow A y),
$$

where $A$ is any sentence of a first-order language for set theory. This principle is almost always omitted from relevant theories, because as Dunn (1987) points out, it has irrelevant instances like $x=y \rightarrow(p \rightarrow p)$. Mares (1992) and then Kremer (1999) have made extensive studies of restricted forms of (1), that preserve relevance, but all that matters for the present is that the unrestricted form has been under scrutiny for a long time now. Bjørdal goes on to use his introduced (1) to prove a result anticipated in the following places, beginning in 1963, Chang (1963), Brady (1971), Gilmore (1974), Jech (1974), White (1979): Petersen (2000, p. 384), Libert (2005, p. 31). In Weber (2010a), exactly the same result as Bjørdal (2010) is again reported and principle (1) is rejected.

What is the justification for not accepting principle (1)? As follows. In any system with naive set comprehension, we will have a Russell set $R=\{x: x \notin x\}$; and given the law of excluded middle, we will have a contradiction $R \in R$ and $R \notin R$. Any solution to the naive set theory problem in this vein will then prove instances of comprehension like

$$
x \in \mu(R) \leftrightarrow R \in R
$$

such that $\forall x(x \in \mu(R) \wedge x \notin \mu(R))$. So there is, inevitably, a sentence $A$ such that $\forall x(A x \wedge \neg A x){ }^{4}$

${ }^{3}$ He adds the definition $x=y:=\forall z(x \in z \leftrightarrow y \in z)$, and calls the immediate consequence (1) full substitution.

4 To take a different sort of example from outside pure set theory, on a paraconsistent account of vagueness, a borderline case of redness will be both red and not red (Weber, to appear). Almost 
How should such information be treated? The question at hand is whether or not a naive set theory should include this principle:

$$
A x, \neg A y \vdash x \neq y .
$$

When $A$ is not restricted in any way, the answer is fairly obviously 'no', for reasons familiar from the intensionality literature. A naive set theory has an undisguised intensional component: sets and properties are being treated as synonymous. Sets are defined by descriptions. But in intensional contexts, (2) is a well-known invalid argument; the failure of nonidentity of discernibles might even be said to define intensionality. Intensionality means our faculty of discernment outstrips the bare structural facts. Descartes doubts his body, does not doubt his mind - and does not, eo ipso, prove that ontological dualism is true. So there is good independent reason to steer clear of (2). ${ }^{5}$

Bjørdal goes on, correctly, to indicate that, "if one understands the extensionality principle as stating that $[\forall z(z \in x \leftrightarrow z \in y) \rightarrow(A x \rightarrow A y)]$, it is easy to show, in light of our result, that all power-sets are paradoxical in the sense that they have members which also fail to be members." Again, the ensuing argument is valid, and the author is correct that it would be unwelcome if all powersets were inconsistent-but he is wrong to give the impression that, according to Weber (2010b), all powersets are inconsistent. We have overtly not taken the extensionality principle to mean that $[\forall z(z \in x \leftrightarrow z \in y) \rightarrow(A x \rightarrow$ $A y)$ ]; in the notation of Weber (2010b), this is just principle (1), and would be a new, added axiom. Principle (1) is not the right way to understand extensionality in this context; again, see Weber (2010a). And for that reason (1) is nowhere to be found in Weber (2010b).

§4. Bjørdal urges that a defined notion of identity may be preferable to a primitive logical notion. We can achieve this without harm for the system of Weber (2010b), following Brady. We define set identity as coincidence of membership:

$$
x=y:=\forall z(z \in x \leftrightarrow z \in y) .
$$

Then the axiom of extensionality follows for free. ${ }^{6}$ We still need to assume some form of substitution, which we do in rule form ${ }^{7}$ :

$$
x=y \vdash \forall z(x \in z \rightarrow y \in z) .
$$

Then the following rule version of Leibniz's law obtains:

$$
x=y \dashv \forall z(x \in z \leftrightarrow y \in z) .
$$

Left to right is by rule (3). Right to left, pick $\{x\}$ for $z$ and we have $x=x \leftrightarrow y=x$. Then modus ponens gives $x=y$. Insofar as any notion of identity "wears its adequacy on its

every object would be a borderline case for some vague predicate; so again there will be some predicate $A$ such that $A x$ and $\neg A x$, for almost every $x$.

5 In the special case that $z \in x$ and $z \notin y$, I have argued that $x \neq y$ should follow Weber (2010a). This is 'downward' or extensional inconsistency, which I do accept, but which is quite distinct from Bjørdal's suggested 'upward' or intensional inconsistency. See Weber (2010a) for detailed discussion on the intensional and extensional properties of a naive set theory.

6 And again, as in footnote 5, the nonidentity of sets that differ with respect to membership also obtains.

7 One could alternatively use an axiom form, as Brady does: $x=y \wedge x=x \rightarrow(A x \rightarrow A y)$. With the extra $x=x$ in the antecedent, contraposition is mooted and so this has the same effect as a noncontraposable rule. 
sleeves" (Bjørdal, 2010), the two-way rule (4) does, but without triggering the fallacious contrapositive consequence that differing in terms of properties implies differing in terms of identity. The system equipped with this much power, but no more, is then capable of supporting a good deal of standard mathematics, as was the burden of Weber (2010b) to show. ${ }^{8}$

The use of modus ponens in the argument just given brings us to our last point. There is no set theory (except for the 'trivial set theory'?) in which every single prima facie plausible looking bit of syntax can be used without some trouble. For those who retain classical logic, there are predicates with extensions that somehow fail to be sets. For those who find set comprehension inalienable, there are logical maneuvers that fail to be valid. To take a particularly striking example, the axiom

$$
\vdash A \wedge(A \rightarrow B) \rightarrow B
$$

cannot be maintained; it is a form of Curry's paradox. At the time of discovery, this led the authors of Meyer et al. (1978) to judge the naive set theory problem impossible. But it turned out that modus ponens is not lost. It is valid in rule form: $A, A \rightarrow B \vdash B$. That is, in effect, why (4) holds over $\vdash$ rather than $\rightarrow$.

Understood as substructural mathematics, this is a discovery about naive sets-for that in inconsistent contexts, modus ponens is an inference, not an axiom. ${ }^{9}$ We now see that this applies to the indiscernibility of identicals, and related notions of identity. The distinction between $\vdash$ and $\rightarrow$ is critical, making possible a solution to the naive set theory problem.

§5. Acknowledgments. Thanks to Graham Priest, Toby Meadows, and Frode Bjørdal for helpful comments on this note. Research funded by ARC Discovery Project Grant 1094962.

\section{BIBLIOGRAPHY}

Bjørdal, F. (2011). The inadequacy of a proposed paraconsistent set theory. Review of Symbolic Logic, 4(1), 106-108.

Brady, R. T. (1971). The consistency of the axioms of the axioms of abstraction and extensionality in a three valued logic. Notre Dame Journal of Formal Logic, 12, 447-453.

Brady, R. T. (1989). The non-triviality of dialectical set theory. In Priest, G., Routley, R., and Norman, J., editors. Paraconsistent Logic: Essays on the Inconsistent. Munich, Germany: Philosophia Verlag, pp. 437-470.

Chang, C. C. (1963). The axiom of comprehension in infinite valued logic. Mathematica Scandinavica, 13, 9-30.

Dunn, J. M. (1987). Relevant predication 1: The formal theory. Journal of Philosophical Logic, 16, 347-381.

8 A nontriviality proof for a very strong paraconsistent set theory may be found in Brady (1989, p. 439) and Priest et al. (1989); notably, this proof covers the 'counterexample' rules $A, \neg B \vdash$ $\neg(A \rightarrow B)$, as well as its contrapositive $A \rightarrow B \vdash \neg A \vee B$. Proving nontriviality for Weber (2010b), in particular a 'counterexample' axiom, has heretofore been an open question and will be addressed elsewhere.

9 As the tortoise might have said to Achilles. 
Gilmore, P. C. (1974). The consistency of partial set theory without extensionality. In Jech, T., editor. Axiomatic Set Theory. Providence American Mathematical Society, pp. $147-153$

Jech, T., editor. (1974). Axiomatic Set Theory. RI: American Mathematical Society.

Kremer, P. (1999). Relevant identity. Journal of Philosophical Logic, 28, 199-222.

Libert, T. (2005). Models for paraconsistent set theory. Journal of Applied Logic, 3, 15-41.

Mares, E. (1992). Semantics for relevant logic with identity. Studia Logica, 51, 1-20.

Meyer, R. K., Routley, R., \& Dunn, M. J. (1978). Curry's paradox. Analysis, 39, 124-128.

Mortensen, C. (1995). Inconsistent Mathematics. Dordrecht: Kluwer Academic Publishers.

Petersen, U. (2000). Logic without contraction as based on inclusion and unrestriced abstraction. Studia Logica, 64, 365-403.

Priest, G., Routley, R., \& Norman, J., editors. (1989). Paraconsistent Logic: Essays on the Inconsistent. Munich, Germany: Philosophia Verlag.

Weber, Z. (2010a). Extensionality and restriction in naive set theory. Studia Logica, 94(1), 87-104.

Weber, Z. (2010b). Transfinite numbers in paraconsistent set theory. Review of Symbolic Logic, 3(1), 71-92.

Weber, Z. (To appear). A paraconsistent model of vagueness. Mind.

White, R. (1979). The consistency of the axiom of comprehension in the infinite valued predicate logic of łukasiewicz. Journal of Philosophical Logic, 8, 503-534.

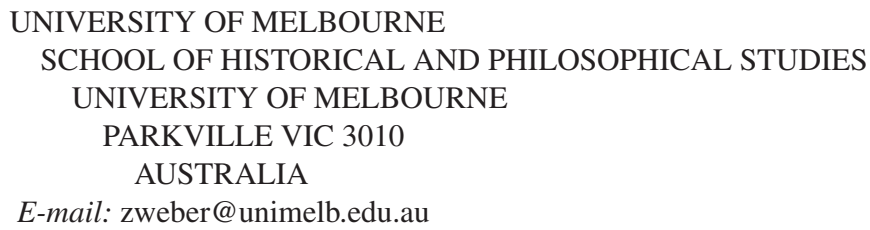

E-mail: zweber@unimelb.edu.au 


\section{University Library}

\section{- M M I N E R VA A gateway to Melbourne's research publications}

Minerva Access is the Institutional Repository of The University of Melbourne

Author/s:

Weber, Z

Title:

THE INADEQUACY OF A PROPOSED PARACONSISTENT SET THEORY REPLY

Date:

2011-03-01

Citation:

Weber, Z. (2011). THE INADEQUACY OF A PROPOSED PARACONSISTENT SET THEORY REPLY. REVIEW OF SYMBOLIC LOGIC, 4 (1), pp.109-113. https://doi.org/10.1017/ S1755020310000304.

Publication Status:

Published

Persistent Link:

http://hdl.handle.net/11343/33009 